\title{
Molecular detection and identification of Wolbachia in three species of the genus Lutzomyia on the Colombian Caribbean coast
}

\author{
Rafael José Vivero ${ }^{1,2,3,4^{*}}$, Gloria Cadavid-Restrepo ${ }^{4}$, Claudia Ximena Moreno Herrera ${ }^{4}$ and Sandra I. Uribe Soto ${ }^{2,3}$
}

\begin{abstract}
Background: The hematophagous habits of insects belonging to the genus Lutzomyia (Diptera: Psychodidae), as well as their role as biological vectors of Leishmania species, make their presence an indication of infection risk. In the present study, seven species of Lutzomyia were identified and screened for natural infections with Wolbachia.

Methods: Collection of sand flies was done in an endemic focus of leishmaniasis on the Colombian Caribbean coast (Department of Sucre, Ovejas municipality). DNA collected from Lutzomyia species was evaluated with PCR for wsp gene amplification to screen for bacterial infection.

Results: Endosymbiotic Wolbachia was found in three species: Lutzomyia c. cayennensis, Lutzomyia dubitans and Lutzomyia evansi. Two Wolbachia strains (genotypes) were found in Lutzomyia spp. These genotypes were previously unknown in dipteran insects. The wLev strain was found in Lutzomyia dubitans, L. c. cayennensis and $L$. evansi and the wLcy strain was found only in L. C. cayennensis.

Conclusions: Genetic analysis indicated that the Wolbachia strains wLcy and wLev belong to the B Supergroup. This study provides evidence of infections of more than one strain of Wolbachia in L. C. cayennensis.
\end{abstract}

Keywords: Wolbachia, Phylogroup wLeva, wsp gene, Lutzomyia, Natural infection

\section{Background}

Los Montes De María is a region located on the Caribbean coast of Colombia which has been historically considered as a focus of several clinical forms of leishmaniasis [1]. In this region, the municipality of Ovejas (Department of Sucre) is of particular epidemiological interest due to the endemic character of leishmaniasis that is occurring in urban, peri-urban and rural areas there. The diversity of Lutzomyia spp. (vector insects) present in Ovejas is high and most of the species are implicated in leishmaniasis transmission $[2,3]$.

In Latin America, vector control campaigns developed for leishmaniasis have mainly focused on chemical control using synthetic pesticides such as pyrethroids and chlorofluazuron [4]. The use of biological alternatives or their derivatives (bacteria, sex pheromones,

\footnotetext{
* Correspondence: rajovigo2001@yahoo.com

'Universidad Nacional de Colombia at Medellín, Medellín, Colombia

${ }^{2}$ Grupo de Investigación en Sistematica Molecular, Universidad Nacional de

Colombia at Medellín, Medellín, Colombia

Full list of author information is available at the end of the article
}

entomopathogenic fungi and toxic plants) have also been considered, but few are used by vector control agencies in Colombia [2]. The medical importance of phlebotomine sand flies (particularly those of the Lutzomyia species) points to the need to consider new and more effective control measures, including some that have already been used for the control of other insects transmitting vector-borne diseases. Among such methods is transfection with bacteria of the genus Wolbachia [5].

Bacteria in the genus Wolbachia are intracellular microorganisms belonging to $\alpha$-proteobacteria (Rickettsia), have maternal inheritance and are commonly found in insect intestines, salivary glands, ovaries and thoraces $[6,7]$. These bacteria may affect the reproductive capabilities of their hosts through diverse mechanisms, generating effects such as the death of male offspring as well as feminization and cytoplasmic incompatibility (CI) [8]. The pathogenic effect of some phenotypes of Wolbachia is now being evaluated on viruses such as Zika, dengue and chikungunya, as well as on Plasmodium $[9,10]$. 
The use of certain strains of Wolbachia is considered to be a promising alternative for decreasing the population density of Lutzomyia species and interfering with the multiplication of parasites and, as a result, Leishmania transmission [11-13]. Thus, initial research efforts have been directed toward screening the presence and circulation of Wolbachia strains in these and other vectors $[14,15]$.

In the Americas, only five species of the genus Lutzomyia have been found to have low levels of Wolbachia infection, with strains belonging to the A and B Supergroups: Lu. cruciata in México, Lu. trapidoi and Lu. vespertilionis in Panamá and Lu. whitmani in Brazil. In Colombia, only $L u$. shannoni was reported as positive for Wolbachia presence [16-18]. Supergroup A, also includes the Wolbachia species detected in Sergentomyia and Phlebotomus [19-21]. Currently, genes (16S $r R N A$, ftsZ, wsp gene) and techniques (Multilocus Sequence Typing technique MLST) are being used to validate the identification and phylogeny of strains of Wolbachia [22].

Partial wsp gene sequences exhibited informative characters useful in the identification of Wolbachia strains detected in Lutzomyia spp. The wsp gene has evolved at a much faster rate than any previously reported gene in Wolbachia [19-22]. Due to this reason, its nucleotide variability facilitates the division into Subgroups and Groups in a consistent manner [22]. The nucleotide variability of the $w s p$ gene and the combination of different primers in PCR reactions is an approach that enables a fast assigning of unknown strains to a particular group, due to its specificity and lack of cross-reactions.

The aim of the present study was molecular detection and identification of the endosymbiont Wolbachia in natural populations of Lutzomyia species found in the municipality of Ovejas on the Colombian Caribbean coast, as well as an analysis of the gene sequence coding for the main surface protein of endosymbiotic Wolbachia (wsp).

\section{Methods}

\section{Phlebotomine survey, processing and identification}

Sand flies were collected in peri-urban environments in the municipality of Ovejas $\left(75^{\circ} 13^{\prime} \mathrm{E} ; 9^{\circ} 31^{\prime} \mathrm{N} ; 277 \mathrm{~m}\right.$ above sea level) during an entomological survey performed between February 21 and 27, 2013. This location is classified as a tropical dry forest ecosystem. Collection was done using CDC white light traps, located indoors and near homes, overnight, between 17:00 and 06:00 h. Shannon traps were also used for collection near homes. Additionally, diurnal collection using a mouth aspirator was done in the vicinity of nocturnal trapping sites. Collected specimens were kept dry in $1.5 \mathrm{ml}$ vials and transported to the laboratory with dry ice. Once at the laboratory, they were kept at $-20{ }^{\circ} \mathrm{C}$. The head and last three abdominal segments were removed from the specimens in order to perform taxonomic identification following the Young \& Duncan classification system [23]. The thorax and remaining abdominal segments were stored at $-20{ }^{\circ} \mathrm{C}$ until DNA extraction.

\section{Pool formation and DNA extraction}

Following taxonomic identification, males and females were separated by species in groups with a variable number of individuals (6 to 10) in $1.5 \mathrm{ml}$ Eppendorf tubes. The formation of groups in this way is justified by differences in the abundance of species in the study area, which complicates statistical interpretation regarding Wolbachia infection rates, but increases the success of molecular detection of bacteria found in natural populations of Lutzomyia in the conditions encountered. In addition, the samples were all collected at the same time.

DNA extraction was done according to the high salt concentration protocol [24]. The quality of DNA (260/ A280 ratio) and concentrations was analysed by Spectrophotometry (Thermo Scientific ${ }^{\mathrm{TM}}$ NanoDrop, Wilmington, USA). Additionally, a partial fragment of the cytochrome $c$ oxidase subunit 1 ( $\operatorname{cox} 1$ ) gene was amplified (Fig. 1) and the spacer region (ITS) between the $23 \mathrm{~S}$ and $16 \mathrm{~S}$ ribosomal gene (Fig. 1), in order to evaluate the quality of DNA present, as well as the absence of PCR inhibitors.

\section{PCR, cloning and DNA fragment sequencing for Wolbachia wsp gene}

Primers $w s p 81 \mathrm{~F}$ (5'-TGG TCC AAT AAG TGA TGA AGA AAC-3') and wsp691R (5'-AAA AAT TAA ACG CTA CTC CA-3') were used to amplify a partial fragment (600 bp) of the gene coding for the main surface protein of endosymbiotic Wolbachia (wsp) (Fig. 1) [25]. The reaction mix used to detect Wolbachia included $80 \mathrm{ng}$ of sample DNA according to the conditions previously described [26, 27]. High fidelity Taq DNA Polymerase (Thermo Scientific, Wilmington, USA) was employed, as well as a conventional thermocycler (BIOMETRA). As a PCR positive control, DNA from ten Aedes (Stegomyia) aegypti larvae (kindly donated by the insectary of the PECET group) infected under laboratory conditions with a reference strain of Wolbachia (Supergroup A, strain $w \mathrm{Mel}$ ) were included (Fig. 1). As a PCR negative control, ultrapure water and DNA of Aedes (= Stegomyia) aegypti without Wolbachia was included (Fig. 1).

Wsp gene amplicons were ligated into JET1.2 vectors (Thermo Scientific) and then transformed into $D H 5 \alpha$ Escherichia coli. At least five independent clones were sequenced for each positive sample involved in detecting Wolbachia strains to generate consensus sequences for further analysis, as well as to mitigate the potential of a mixed infection in the pools [27]. Clones with the partial 

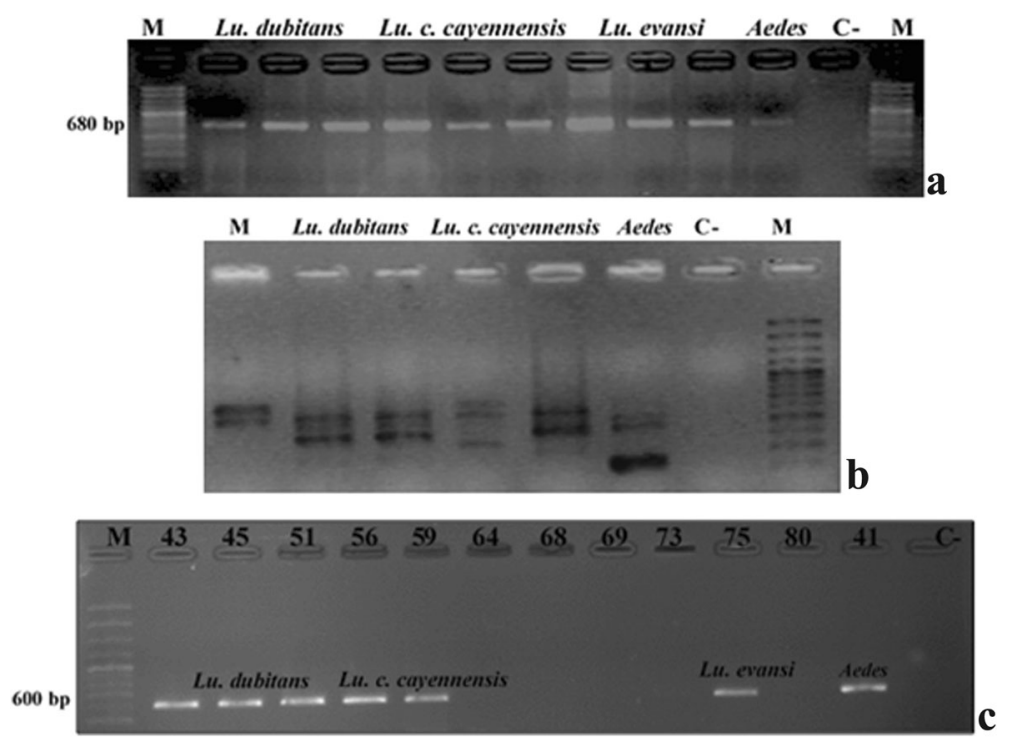

Fig. 1 PCR from Lutzomyia genomic DNA pools. a PCR amplification of the cox1 gene fragment to evaluate the quality of DNA and absence of inhibitors from genomic DNA pools. b PCR for ITS to estimate the quality of available bacterial DNA. c PCR amplification from a partial fragment of wsp gene with primers wsp in different species of Lutzomyia. PCR products were evaluated in $1 \%$ electrophoresis gels. Abbreviations: M, a 100 bp DNA ladder; C-, negative control

products of $w s p$ were verified by sequencing in both directions using universal primers from Macrogen Inc., Korea. For each assay, a negative control (no DNA) as well as a positive control (control PCR product by the cloning kit) was included.

\section{Identity of Wolbachia strains and their positions in phylogroups}

The wsp gene obtained from Wolbachia were sent for sequencing (Macrogen, Korea) and the results were compared to previously identified sequences using the basic local alignment search tool (BLASTN) (https:// www.ncbi.nlm.nih.gov/) and edited with Bioedit v.7.2.5 [28] in order to obtain detected consensus sequence for every Lutzomyia species. This was also made with gene sequences of Wolbachia, which were available in the National Center for Biotechnology Information (NCBI) database and Wolbachia MLST database (http://pubmlst.org/wolbachia/). The nucleotide alignment reading framework reported by O'Neill (ftp://ftp.ebi.ac.uk/pub/ databases/embl/align/; Access Number DS42468) was considered, which suggests starting the analysis by translating the sequences to amino acids as a guide to align the DNA sequences of the wsp gene [27].

Alignments of sequences of $w s p$ genes obtained in Lutzomyia and reported in GenBank (Additional file 1) were performed using the Clustal W and Muscle algorithms incorporated in MEGA 6. Verification of recombination events and the presence of chimeras was performed with RDP4 (Recombination Detection Program version 4) software, using all sequences of $w s p$ obtained in this study in order to ensure the accuracy of nucleotide variability with respect to previously reported sequences in GenBank (Additional file 1). Patterns of genetic divergence (nucleotide composition, number of haplotypes, variable sites) and K2P genetic distances were evaluated using Bioedit v.7.2.5 and DNAsp 5.0 software.

All aligned sequences (= haplotypes) of wsp genes obtained in this study and reported in GenBank were exported using MEGA software. Description codes include the following abbreviations for species: Lev, Lutzomyia evansi; Lcy, Lutzomyia c. cayennensis and Luduv, Lutzomyia dubitans followed by the letters ov, which refer to the place where they were collected in Colombia (ov, municipality of Ovejas) and numbers corresponding to specimens with the same sequence.

Subsequently, the identities and relationships of the Wolbachia strains obtained in our study was determined by performing a phylogenetic inference analysis using the Bayesian method (number of generations $=1,000,000$ ) with the MrBayes 3.0 software under the substitution model GTR + G (number of estimated parameters $k=139$; Akaike information criterion $($ AIC $)=7807.8819)$; with jModeltest 2.1.4 software [29]; and Phyml 3.0 software [30]. All of the sequences obtained in the present study (KR907869-KR907874) were submitted to GenBank (Additional file 1).

\section{PCR amplification of the HSP-70 N Leishmania gene in female groups}

A PCR test was done to screen Leishmania infection in females of Lutzomyia. The primers used were HSP70- 
F25 (5'-GGA CGC CGG CAC GAT TKC T-3') and HSP70-R617 (5'-CGA AGA AGT CCG ATA CGA GGG A-3'), which amplify a 593 bp partial segment of the HSP-70 N gene (coding for Heat shock protein 70) [31]. PCR testing was done following the conditions and thermal profile described by Fraga et al. [31]. As a positive control, DNA from Leishmania panamensis (reference strain UA140) and Leishmania braziliensis (reference strain UA 2903), which was kindly provided by the PECET group of the Universidad de Antioquia, was included.

\section{Results}

\section{Taxonomic identification of sand flies}

A total of 325 individuals were collected from peri-urban environments. Morphological and taxonomic guides allowed the identification of seven species: $L u$. evansi, $L u$. trinidadensis, Lu. c. cayennensis, Lu. dubitans, Lu. gomezi, $L u$. rangeliana and $L u$. atroclavata (Table 1). Lutzomyia dubitans (110 specimens; 33.8\%) and Lu. c. cayennensis (107 specimens; 32.2\%) were the species found in the highest proportions (Table 1). Thirty-five pools were formed according to sex and taxonomic assignation as described above.

\section{Wolbachia (wsp gene) infection}

As expected, all PCR fragments of the $w s p$ gene were approximately $600 \mathrm{bp}$ in size, and were obtained from three species: $L u$. dubitans, $L u$. c. cayennensis and $L u$. evansi. Among these three sand fly species, seven pools were positive for Wolbachia (Fig. 1, Table 1). Low relative infection rates were found in $L u$. dubitans and $L u$. $c$. cayennensis ( 3 positive pools; $8.5 \%$ for both species) (Table 1). In Lu. evansi (1 positive pool; $2.8 \%$ ), only one group was positive. It worth noting that Wolbachia was present in both sexes of Lutzomyia, particularly in $L u$. dubitans (males, 5.7\%; females, 2.8\%) and Lu. c. cayennensis (males, 5.7\%; females, $2.8 \%$ ) (Table 1), while in $L u$. evansi Wolbachia was detected only in males. Lutzomyia rangeliana, Lu. trinidadensis, Lu. gomezi and Lu. atroclavata were all negative for Wolbachia. The positive control strain $w \mathrm{Mel}$ successfully amplified in all PCR assays of the wsp gene for Wolbachia and the negative controls showed no PCR products.

\section{Wolbachia identity based on comparisons with previous sequences and assignation of phylogroups using wsp gene sequences}

Based on DNA sequences, the presence and identity of Wolbachia in Lu. dubitans, Lu. evansi and Lu. c. cayennensis was determined. Nucleotide variability analysis based on fragments of $523 \mathrm{bp}$, showed only 15 variable sites among wsp sequences of Wolbachia obtained from Lutzomyia species (Fig. 2). In the Bayesian inference, 59 partial sequences of the Wolbachia wsp gene were included from strains related to arthropods, which are located in supergroups A and B, representing 24 groups with 57 previously detected strains from a wide number of insects (Additional file 1). Five haplotypes (HP) of the wsp gene (HP1 to HP5) were found in this study, which were described with short codes that allow the location of Wolbachia genotypes to be determined in relation to the species in which they were detected and that facilitate locating them in the tree created with all the sequences by Bayesian inference (Fig. 3).

The five haplotypes HP1 (WbLevov75); HP2 (WbLcyov56/WbLdubov45); HP3 (WbLcyov59); HP4 (WbLdubov43); and HP5 (WbLdubov51) differed due to 2-11 insertion-deletion and point mutation events

Table 1 Formation of pools of Lutzomyia spp. for detection of infection by Wolbachia in the peri-urban environments in the municipality of Ovejas, Department of Sucre, Colombia

\begin{tabular}{|c|c|c|c|c|}
\hline Species & Sex & $\begin{array}{l}\text { No. of pools formed } \\
\text { (No. of specimens) }\end{array}$ & $\begin{array}{l}\text { No of positive pools } \\
\text { with Wolbachia (\%) }\end{array}$ & $\begin{array}{l}\text { Total no. of specimens } \\
\text { per species analysed (\%) }\end{array}$ \\
\hline \multirow[t]{2}{*}{ Lu. dubitans } & Female & $8(80)$ & $2(5.7)$ & $110(33.8)$ \\
\hline & Male & $3(30)$ & $1(2.8)$ & \\
\hline \multirow[t]{2}{*}{ Lu.c. cayennensis } & Female & $4(40)$ & $1(2.8)$ & 107 (32.2) \\
\hline & Male & $7(67)$ & $2(5.7)$ & \\
\hline \multirow[t]{2}{*}{ Lu. gomezi } & Female & $3(24)$ & 0 & $41(9.5)$ \\
\hline & Male & $2(17)$ & 0 & \\
\hline \multirow[t]{2}{*}{ Lu. trinidadensis } & Female & $1(7)$ & 0 & $33(10.1)$ \\
\hline & Male & $3(26)$ & 0 & 1 \\
\hline \multirow[t]{2}{*}{ Lu. rangeliana } & Female & $1(10)$ & 0 & $16(4.9)$ \\
\hline & Male & $1(6)$ & 0 & \\
\hline Lu. evansi & Male & $1(8)$ & $1(2.8)$ & $8(2.4)$ \\
\hline Lu. atroclavata & Female & $1(10)$ & 0 & $10(3)$ \\
\hline Total & - & 35 (325) & $7(20)$ & $325(100)$ \\
\hline
\end{tabular}




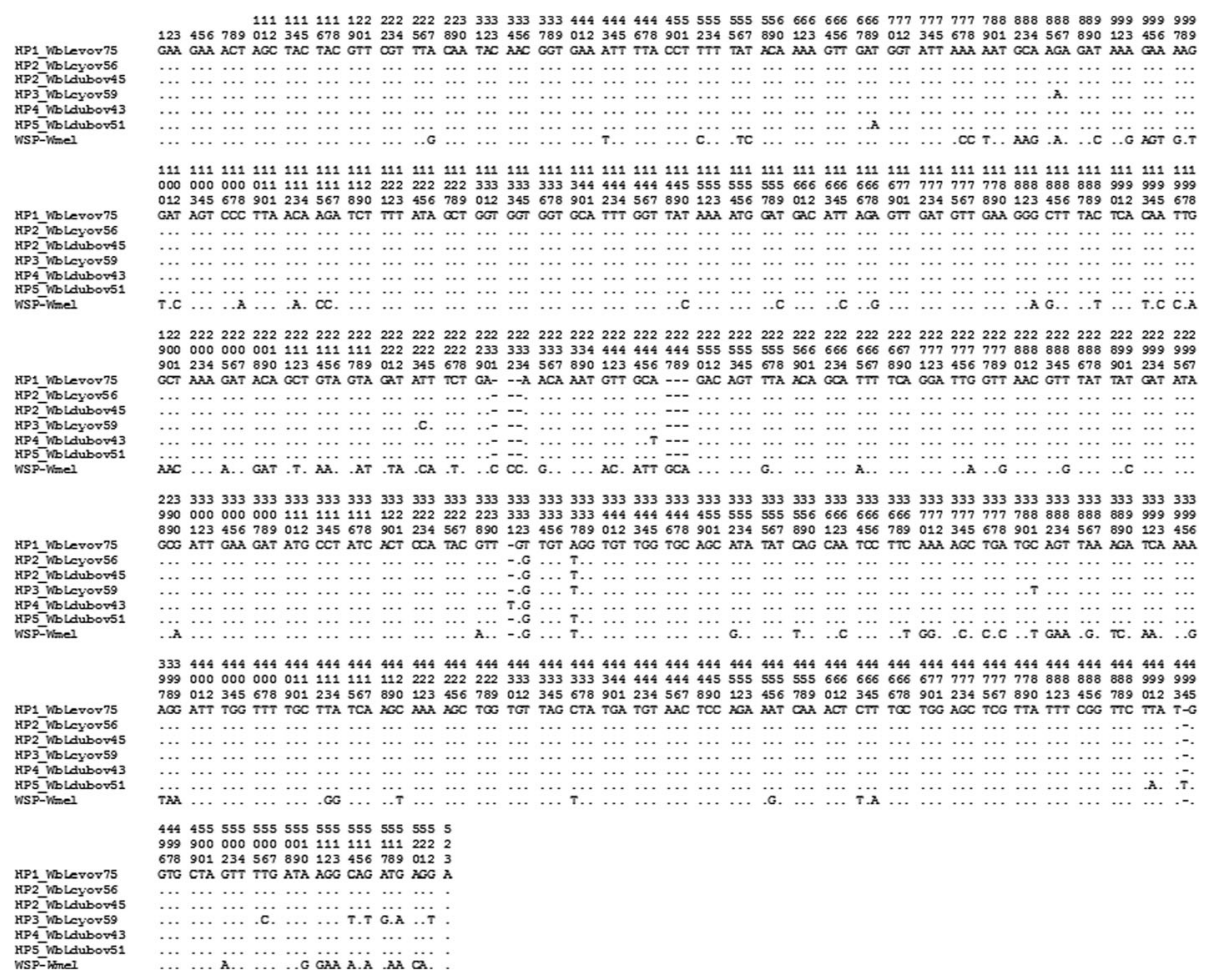

Fig. 2 Multiple alignment of partial nucleotide sequences of wsp gene (Positions 1-523) of Wolbachia strains, detected in Lutzomyia species (blue) collected in Ovejas (Sucre, Colombia). Description codes include the following abbreviations for species: Lev, Lutzomyia evansi; Lcy, Lutzomyia c. cayennensis, and Luduv, Lutzomyia dubitans followed by ov, referring to the place of collection in Colombia (ov, municipality of Ovejas) and numbers corresponding to specimens with the same sequence. The haplotypes are: HP1, WbLevov75; HP2, WbLcyov56WbLdubov45; HP3, WbLcyov59; HP4, WbLdubov43; and HP5, WbLdubov51. strain WMel is the positive control

(Fig. 2). The values of Kimura 2-parameter pairwise genetic distances among the haplotypes of Wolbachia were between 0.004 and 0.021 (Table 2), suggesting the existence of different strains. The haplotypes WbLevov75, WbLcyov56, WbLdubov45, WbLdubov51 and WbLdubov43; representing the $w L e v$ strain, showed low levels of genetic differentiation (0.004) and high similarity (99.6\%) (Table 2).

The haplotype WbLcyov59-HP3, representing the wLcy strain, exhibited similarity of $97.9 \%$ and higher values for genetic distances (0.017-0.021) when compared with haplotypes of the wLev strain (Table 2). The wLev strain was present in Lu. c. cayennensis, Lu. dubitans and Lu. evansi, but in $L u$. c. cayennensis the $w L c y$ strain was also detected.

The wLev strain (Table 2), showed low levels of genetic differentiation as compared to the strains from Supergroup B, as well as showing affinity for strains from the groups Unif (wUnif $=0.017-0.026$; wInd $=$ $0.014-0.019)$, Con ( $w$ Sit $=0.020-0.026 ; \quad w$ Con $=0.022-$ $0.027 ; w G e l=0.014-0.019 ; w S t r i=0.019-0.024)$ and Per (wPer $=0.014-0.020)$ (Table 2). In contrast, high values of genetic distances $(0.234-0.255)$ were found by comparing the strains clustered into the Supergroup A when wNiv from Aedes (Stegomyia) niveus was included (Table 2).
The haplotype $w L c y$ showed low genetic distance values in comparison to strains located in Subgroup B, such as Prn $(w \operatorname{Prn}=0.017)$ (Table 2) identified in the phlebotomine Phlebotomus perniciosus; strains wInd, wUnif (Group Unif $=0.019$ ) out of group Unif; strains wSit (0.024), wCon (0.027) wGel (0.019), wStri (0.019) out of group Con, detected in mosquito species Mansonia indiana, Mansonia uniformis and Culex gelidus; and in the homopteran Laodelphax striatellus, respectively (Table 2, Additional file 1). Both wLev and wLcy showed higher values of genetic distances in relation to Wolbachia strains in Supergroup A, among which wNiv (0.240), $w \mathrm{~Pa}$ (0.230) and wSub (0.231) are highlighted.

The percentage divergence based on alignment, which includes a large number of available sequences, suggests that wsp gene sequences from Wolbachia present considerable intra- and inter-genic variation. This can be summarized as follows: between sequences of the same strain there is $0.4 \%$ variation; between strains of the same group there is $1-2.1 \%$ variation; between strains of different groups located in the same supergroup there is $1.9-2.7 \%$ variation; and between strains of different supergroups there is $13.4-25.5 \%$ variation (Table 2 ). These percentages are consistent with the established ranges 


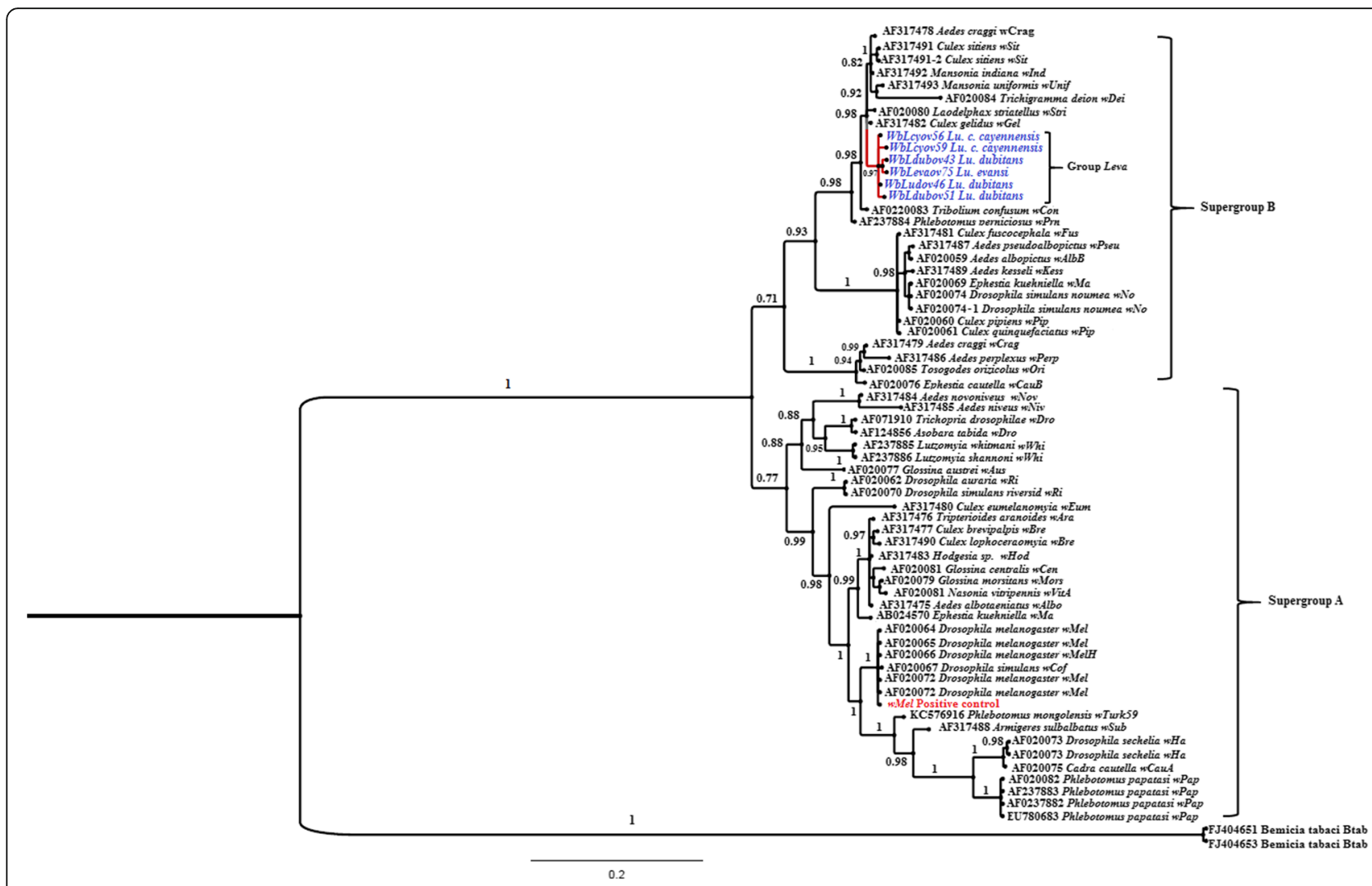

Fig. 3 Phylogenetic relationships of Wolbachia strains inferred using wsp gene including the ones detected in Lutzomyia species (blue) collected in Ovejas (Sucre, Colombia). Numbers in nodes represent Bayesian posterior probabilities. Reconstruction performed with MrBayes (version 3.0). The wMel positive control is indicated in red

Table 2 Values of genetic distances K2P and percent of sequence identity based on alignment of the wsp gene among strains of Wolbachia in the Leva, Con, Unif and Pern groups (Supergroup B) and some strains (WNiv and wWhi) of Supergroup A

\begin{tabular}{|c|c|c|c|c|c|c|c|c|c|c|c|}
\hline \multirow{3}{*}{$\begin{array}{l}\text { Groups } \\
\text { Strain }\end{array}$} & \multicolumn{9}{|l|}{ Supergroup B } & \multicolumn{2}{|c|}{ Supergroup A } \\
\hline & \multicolumn{2}{|l|}{ Leva } & \multicolumn{2}{|l|}{ Unif } & \multicolumn{4}{|l|}{ Con } & \multirow{2}{*}{$\begin{array}{l}\text { Per } \\
\text { wPer }\end{array}$} & \multirow{2}{*}{$\begin{array}{l}\mathrm{Niv} \\
\text { WNiv }^{(\%)}\end{array}$} & \multirow{2}{*}{$\begin{array}{l}\text { Whi } \\
\text { wWhi }\end{array}$} \\
\hline & $w$ Lev $^{(\%)}$ & $w L C y^{(\%)}$ & wUnif ${ }^{(\%)}$ & wind & $\mathrm{wSit}^{(\%)}$ & wCon & wGel & wStri & & & \\
\hline wLev & $0.004^{99.6}$ & & & & & & & & & & \\
\hline$w$ Lcy & $0.017-0.021^{97.9}$ & - & & & & & & & & & \\
\hline wUnif & $0.017-0.026^{97.4}$ & $0.019^{98.1}$ & - & & & & & & & & \\
\hline wind & $0.014-0.019^{98.1}$ & $0.019^{98.1}$ & $0.010^{99.0}$ & - & & & & & & & \\
\hline wSit & $0.020-0.026^{97.4}$ & $0.024^{97.6}$ & 0.014 & 0.014 & - & & & & & & \\
\hline wCon & $0.022-0.027^{97.3}$ & $0.027^{97.6}$ & $0.022^{97.8}$ & 0.012 & $0.017^{98.3}$ & - & & & & & \\
\hline wGel & $0.014-0.019^{98.1}$ & $0.019^{98.1}$ & 0.014 & 0.014 & 0.010 & 0.012 & - & & & & \\
\hline wStri & $0.019-0.024^{97.6}$ & $0.019^{98.1}$ & 0.010 & 0.019 & 0.015 & 0.010 & 0.016 & - & & & \\
\hline wPer & $0.014-0.020^{98}$ & $0.017^{98.3}$ & 0.017 & 0.027 & $0.022^{97.8}$ & 0.017 & 0.014 & 0.022 & - & & \\
\hline wNiv & $0.234-0.255^{74.5}$ & $0.240^{76}$ & $0.229^{77.1}$ & 0.216 & $0.223^{77}$ & 0.226 & 0.222 & 0.219 & $0.219^{78.1}$ & - & \\
\hline wWhi & $0.174-0.178^{86.6}$ & $0.167^{83.3}$ & $0.183^{81.7}$ & 0.176 & $0.173^{82.7}$ & 0.179 & 0.173 & 0.176 & $0.173^{82.7}$ & $0.136^{86.4}$ & - \\
\hline
\end{tabular}

Note: The superscripts indicate the percent similarity between the sequences and were determined only among some strains representing different levels of variation: within the same strain, between strains of the same group, between strains of different groups, among strains from different supergroups 
for the separation of strains and current assignment of Wolbachia groups [27].

Phylogenetic relationships estimated by Bayesian Inference analysis (including $449 \mathrm{bp}$ in the final alignment) grouped the strains $w$ Lev and $w L c y$ in a new group called "wLeva" (branch support of 0.97), located in the Supergroup B, and based on the robustness of clade posterior probability (0.71) with respect to Supergroup A (Fig. 3). The Leva group has a close phylogenetic relationship (0.98) with the Dei, Crag, Unif, and Prn groups (Fig. 3).

\section{Leishmania infection}

Eighteen female groups composed of 171 individual specimens of Lu. evansi, Lu. dubitans, Lu. c. cayennensis, $L u$. gomezi, Lu. trinidadensis, $L u$. rangeliana and $L u$. atroclavata, were negative for Leishmania infection.

\section{Discussion}

This study reports a natural infection of endosymbiotic Wolbachia in natural populations of $\mathrm{Lu}$. dubitans, $\mathrm{Lu}$. c. cayennensis and $\mathrm{Lu}$. evansi for the first time from the peri-urban environment of a leishmaniasis focus transmission on the Caribbean coast of Colombia.

Different studies with similar sample sizes (between 141 and 547 individuals) and grouping of individuals by species (10-100) have been developed, and determine infection rates [32]. We decided not to do calculations infection rates from DNA Lutzomyia groups because we consider that the prevalence of Wolbachia may be low and poorly estimated. For this reason, we only emphasize on infected species and characterization of genetic haplotypes.

Lutzomyia evansi and Lu. dubitans were found to be infected with Wolbachia by a strain named wLev, while $L u$. c. cayennensis was infected with both strains of Wolbachia (wLcy and wLev). This is consistent with the presence of these insect species in a uniform ecological region (similar collection localities). Regarding $L u$. c. cayennensis, there exists a possibility that Wolbachia infected this species more than once, which would explain the presence of two different strains. In some studies, some Wolbachia strains belonging to different subgroups or groups have been observed to infect the same host species [33].

The groupings based on Wolbachia wsp gene sequences included in this study were well supported and consistent with those previously reported for Supergroups A and B [34]. The Wolbachia strains wLev and $w L c y$ reported in this study appear to be included as a group in Supergroup B, which is common in arthropods. Wolbachia strains wLev and wLcy show close relationships to the Prn, Con and Unif groups of Supergroup B [12]. Proximity to the group Prn is highlighted, because the wPrn strain was found in the host Ph. pernisiosus [12]. In contrast, strains $w L c y$ and wLev located in this group do not appear to show a close relationship to Wolbachia strains in group Whi ( $L u$. whitmani and $L u$. shannoni), which are detected in species of the subfamily Phlebotominae, even though they have a closely related host and a similar continental distribution [23]. Interestingly, some strains of Supergroup B (wPip, WBoL and $w V u l$ ) have phenotypes associated with feminization of males, as well as mortality and cytoplasmic incompatibility [35]. Each of these reproductive alterations are advantageous to Wolbachia as they are correlated to an increase in infected females. This group of strategies is called reproductive parasitism [36].

The species $L u$. evansi, Lu. dubitans and Lu. c. cayennensis were found positive for Wolbachia infection both by PCR and by sequencing of the $w s p$ gene, that enables a fast assigning of unknown strains to a particular group [37]. These three species have a history of natural infection by species of Leishmania [1, 3]. However, in this study, Leishmania was not detected in them. The prevalence of natural infections with Leishmania in sand flies is low. The process of simultaneous identification of Leishmania and Wolbachia can be complicated and needs to be initially standardized under laboratory conditions. Other researchers have reported differences in the sensitivity of different molecular markers and conventional tests (PCR, RFLP, isozyme patterns, hybridization with DNA probes) for the detection, diagnosis and identification of Leishmania species [37]; and they propose that exploring the possibility of viewing promastigotes by the dissection of digestive tracts and the implementation of more variants of $\mathrm{PCR}$ with genus-specific primers would be beneficial. Also it is necessary to indicate that the absence of Wolbachia and Leishmania in Lutzomyia species may be influenced by the sampling scheme (spot scouting) and the size of the analyzed sample, which reduces the possibility of detecting positive DNA of Leishmania. Identification of species of Leishmania from vectors has also been constrained by the need to isolate the parasite from one or more of the small proportion of sand flies that are normally found to be infected, ranging from 0.001 to $2.26 \%$ for Leishmania transmission [37].

It is desirable to advance our understanding of the biology and spread of Wolbachia bacteria in relation to Leishmania infection, given the fact that different studies show the impact of these bacteria in host-parasite interactions with a potential use in reducing the risk of infectious diseases caused by parasites and transmitted to humans by insects [38]. Many invertebrates are infected by Wolbachia, and the bacteria's success may be credited to the diverse phenotypes (mutualism or reductive parasitism) that result from infection. The persistence of 
the Wolbachia infections and phenotype estimation in natural populations of Lutzomyia in the municipality of Ovejas, are determinants to make strong correlations of the role of Wolbachia on the development of Leishmania. Another area of study, may include the introduction of Wolbachia in Lutzomyia evansi (main vector and abundant species in the Caribbean coast) and its interaction with Leishmania.

Additionally, it has been found that the presence of some strains of Wolbachia in mosquitoes can regulate the expression of genes involved in the immune responses, resulting in inhibition of the replication, multiplication, or resistance to the proliferation of viruses, parasites, and microfilariae [39]. In this sense, Wolbachia can also be visualized as a microorganism for biological control, that is based on the substitution of the microbiome of the vector by microorganisms that affect vector's pathogen load. Replacement microbiota may represent unmodified microbial species that normally do not colonize a particular vector species, or genetically engineered symbiotic bacteria [40]. A vector's microbiome can be altered either through the stable "conversion" of vector populations in the wild or by introducing the desirable microbiota through bait stations [40,41], which allows for a continuous modification of vector populations.

\section{Conclusions}

Our study represents a significant advance in the understanding of natural infections of Wolbachia in Lutzomyia. Further studies are needed to investigate the dynamics of infections with Wolbachia and Leishmania in natural populations of Lutzomyia present in other areas of leishmaniasis transmission.

\section{Additional file}

Additional file 1: Nomenclature of Wolbachia supergroups, groups, strains used and host insects related. These strains were used to compare genetic distances and perform phylogenetic reconstructions. (DOCX 20 kb)

\section{Abbreviations \\ cox1: Cytochrome c oxidase subunit 1; Le: Leishmania; Lu: Lutzomyia; wsp: Gene coding for the main surface protein of endosymbiotic Wolbachid}

\section{Acknowledgements}

We are grateful for the support given by members of the Program of Study and Control of Tropical Diseases (PECET) of the University of Antioquia and the Biomédicas Research Group of the University of Sucre in Sincelejo, Colombia, during this entomological survey. We thank the different visited communities in the municipality of Ovejas (Department of Sucre) during our surveys for giving us access to their facilities, providing hospitality and collaborating with fieldwork. We are also grateful for the in-field sampling help of Horacio Cadena, researcher of the Program for Study and Control of Tropical Diseases of the University of Antioquia, as well as Luis Estrada and Edgar Ortega, researcher of the Biomédicas Research Group of the University of Sucre, Sincelejo.

\section{Funding}

This project is part of the activities associated with a consortium financed by Administrative Department of Science, Technology and Innovation-COLCIENCIAS (Grant CT-695-2014 and Doctoral studies in Colombia 528-2011). The funders had no role in the study design, data collection and analysis, decision to publish or preparation of the manuscript.

\section{Availability of data and materials}

The datasets supporting the conclusions of this article are included within the article and its additional file. The newly-generated sequences are submitted to the GenBank database under accession numbers KR907869-KR907874.

\section{Authors' contributions}

RJV: Designed the study, performed the experiments and field work, analyzed the data and contributed to writing the manuscript. CXMH, GECR and SUS: Designed the study, analyzed the data and contributed to writing the manuscript. All authors read and approved the final manuscript.

\section{Competing interests}

The authors declare that they have no competing interests.

\section{Consent for publication}

Not applicable.

\section{Ethics approval and consent to participate}

Sand fly collection was performed in accordance with the parameters of Colombian decree number 1376 , which regulates specimen collection of biologically diverse wild species for non-commercial research. No specific permits were required for this study. The sand flies were collected on private property and permissions were received from landowners prior to sampling.

\section{Author details}

${ }^{1}$ Universidad Nacional de Colombia at Medellín, Medellín, Colombia. ${ }^{2}$ Grupo de Investigación en Sistematica Molecular, Universidad Nacional de Colombia at Medellín, Medellín, Colombia. ${ }^{3}$ PECET (Programa de Estudio y Control de Enfermedades Tropicales), Universidad de Antioquia, Medellín, Colombia. ${ }^{4}$ Grupo de Microbiodiversidad y Bioprospección, Laboratorio de Biología Celular y Molecular, Universidad Nacional de Colombia at Medellín, Medellín, Colombia.

Received: 16 July 2016 Accepted: 10 February 2017

Published online: 28 February 2017

\section{References}

1. Cortés A, Pérez-Doria A, Bejarano E. Flebotomíneos (Diptera: Psychodidae) antropofílicos de importancia en salud pública en Los Montes de María. Rev Cuba Med Trop. 2009:61:220-5.

2. Bates PA, Depaquit J, Galati EAB, Kamhawi S, Maroli M, Mcdowell MA, et al. Recent advances in phlebotomine sand fly research related to leishmaniasis control. Parasit Vectors. 2015:8:131.

3. Cochero BS, Anaya EY, Díaz EY, Paternina EM, Luna EA, Luis E. Infección natural de Lutzomyia cayennensis cayennensis con parásitos tripanosomatídeos (Kinetoplastida: Trypanosomatidae) en Los Montes de María, Colombia. Rev Cuba Med Trop. 2007;59:35-9.

4. Sharma U, Singh S. Insect vectors of Leishmania: distribution, physiology and their control. J Vector Borne Dis. 2008;45:255-72.

5. Jeffries $\mathrm{CL}$, Walker T. Wolbachia biocontrol strategies for arboviral diseases and the potential influence of resident Wolbachia strains in mosquitoes. Curr Trop Med Reports. 2016;3:20-5.

6. Serbus LR, Casper-Lindley C, Landmann F, Sullivan W. The genetics and cell biology of Wolbachia-host interactions. Annu Rev Genet. 2008;42:683-707.

7. Rossi P, Ricci I, Cappelli A, Damiani C, Ulissi U, Mancini MV, et al. Mutual exclusion of Asaia and Wolbachia in the reproductive organs of mosquito vectors. Parasit Vectors. 2015;8:1-10.

8. Blagrove MS, Arias-Goeta C, Failloux AB, Sinkins SP. Wolbachia strain wMel induces cytoplasmic incompatibility and blocks dengue transmission in Aedes albopictus. Proc Natl Acad Sci USA. 2012;109:255-60.

9. Moreira LA, Iturbe-Ormaetxe I, Jeffery JA, Lu G, Pyke AT, Hedges LM, et al. A Wolbachia symbiont in Aedes aegypti limits infection with dengue, chikungunya and Plasmodium. Cell. 2009;139:1268-78. 
10. Hughes $G L$, Koga R, Xue P, Fukatsu T, Rasgon JL. Wolbachia infections are virulent and inhibit the human malaria parasite Plasmodium falciparum in Anopheles gambiae. PLoS Pathog. 2011;7:e1002043.

11. Matsumoto K, Izri A, Dumon H, Raoult D, Parola P. First detection of Wolbachia spp., including a new genotype, in sand flies collected in Marseille, France. J Med Entomol. 2008:45:466-9.

12. Ono M, Braig HR, Munstermann LE, Ferro C, O'Neill SL. Wolbachia infections of phlebotomine sand flies (Diptera: Psychodidae). J Med Entomol. 2001;38:237-41.

13. Azpurua J, De La Cruz D, Valderama A, Windsor D. Lutzomyia sand fly diversity and rates of infection by Wolbachia and an exotic Leishmania species on Barro Colorado Island, Panama. PLoS Negl Trop Dis. 2010;9(4):3.

14. Hughes GL, Rasgon JL. Transinfection: A method to investigate Wolbachiahost interactions and control arthropod-borne disease. Insect Mol Biol. 2014;23(2):141-51.

15. Brownstein JS, Hett E, O'Neill SL. The potential of virulent Wolbachia to modulate disease transmission by insects. J Invertebr Pathol. 2003;84:24-9.

16. Parvizi $P$, Bordbar A, Najafzadeh N. Detection of Wolbachia pipientis, including a new strain containing the wsp gene, in two sister species of Paraphlebotomus sand flies, potential vectors of zoonotic cutaneous leishmaniasis. Mem Inst Oswaldo Cruz. 2013;108:1-7.

17. Mirkery-Pachecho O, Marina C, Ibañez B, Sanchez D, Castillo V. Infeccion natura de Lutzomyia cruciata (Diptera: Psychodidae, Phlebotominae) con Wolbachia en cafetales de Chiapas, México. Act Zoológica Mex. 2012;8(2):401-13.

18. Island C, Azpurua J, Cruz DD L, Valderama A, Windsor D. Lutzomyia sand fly diversity and rates of infection by Wolbachia and an exotic Leishmania species on Barro. 2010. p. 4

19. Bordbar A, Soleimani S, Fardid F, Zolfaghari MR, Parvizi P. Three strains of Wolbachia pipientis and high rates of infection in Iranian sandfly species. Bull Entomol Res. 2014;104:195-202.

20. Parvizi P, Benlarbi M, Ready PD. Mitochondrial and Wolbachia markers for the sandfly Phlebotomus papatasi: Little population differentiation between peridomestic sites and gerbil burrows in Isfahan province. Iran Med Vet Entomol. 2003;17:351-62.

21. Kassem HA, Osman G. Maternal transmission of Wolbachia in Phlebotomus papatasi (Scopoli). Ann Trop Med Parasitol. 2007;101:435-40.

22. Baldo L, Hotopp JCD, Jolley KA, Bordenstein SR, Biber SA, Choudhury RR, et al. Multilocus sequence typing system for the endosymbiont Wolbachia pipientis. Appl Environ Microbiol. 2006;72:7098-110.

23. Young DG, Duncan M. Guide to the identification and geographic distribution of Lutzomyia sand flies in Mexico, the West Indies, Central and South America. J Biol Sci. 1994

24. Golczer G, Arrivillaga J. Modificación de un protocolo estándar de extracción de ADN para flebotominos pequeños (Phlebotominae: Lutzomyia). Rev Colomb Entomol. 2008;34:199-202.

25. Braig HR, Zhou W, Dobson SL, O'Neill SL. Cloning and characterization of a gene encoding the major surface protein of the bacterial endosymbiont Wolbachia pipientis. J Bacteriol. 1998;180:2373-8.

26. Werren JH, Zhang W, Guo LR. Evolution and phylogeny of Wolbachia: reproductive parasites of arthropods. Proc R Soc B. 1995;261:55-71.

27. Zhou W, Rousset F, O'Neil S. Phylogeny and PCR-based classification of Wolbachia strains using wsp gene sequences. Proc Biol Sci. 1998:265:509-15.

28. Hall T. BioEdit: a user-friendly biological sequence alignment editor and analysis program for Windows 95/98/NT. Nucleic Acids Symp Ser. 1999;41:95-8.

29. Darriba D, Taboada G, Doallo R, Posada D. jModelTest 2: more models, new heuristics and parallel computing. Nat Methods. 2012;9(8):772.

30. Guindon S, Dufayard J, Lefort V, Anisimova M, Hordijk W, Gascuel O. New algorithms and methods to estimate maximum-likelihood phylogenies: assessing the performance of PhyML 3.0. Syst Biol. 2010;59(3):307-21.

31. Fraga J, Montalvo AM, De Doncker S, Dujardin J-C, Van der Auwera G. Phylogeny of Leishmania species based on the heat-shock protein 70 gene. Infect Genet Evol. 2010;10:238-45.

32. Marcon HS, Coscrato VE, Selivon D, Perondini AL, Marino CL. Variations in the sensitivity of different primers for detecting Wolbachia in Anastrepha (Diptera: Tephritidae). Brazilian J Microbiol. 2011:42:778-85.

33. Kondo N, Shimada M, Fukatsu T. Infection density of Wolbachia endosymbiont affected by co-infection and host genotype. Biol Lett. 2005;1:488-91.

34. Hinrich J, Schulenburg GVD, Hurst GDD, Huigens TME, Van Meer MMM, Jiggins FM, et al. Molecular evolution and phylogenetic utility of Wolbachia $\mathrm{ftsZ}$ and wsp gene sequences with special reference to the origin of malekilling. Mol Biol Evol. 2000;17:584-600.
35. Jiggins FM, Bentley JK, Majerus ME, Hurst GD. How many species are infected with Wolbachia? Cryptic sex ratio distorters revealed to be common by intensive sampling. Proc Biol Sci. 2001;268:1123-6.

36. Zug R, Hammerstein P. Bad guys turned nice? A critical assessment of Wolbachia mutualisms in arthropod hosts. Biol Rev. 2014

37. Pintureau $B$, Chaudier $\mathrm{S}$, Lassabliere $F$, Charles $H$, Grenier S. Addition of wsp sequences to the Wolbachia phylogenetic tree and stability of the classification. J Mol Evol. 2000;51:374-7.

38. Slatko BE, Luck AN, Dobson SL, Foster JM. Wolbachia endosymbionts and human disease control. Mol Biochem Parasitol. 2014; 88-95

39. LePage D, Bordenstein SR. Wolbachia: Can we save lives with a great pandemic? Trends Parasitol. 2013;29(8):385-93.

40. Leitner WW, Wali T, Kincaid R, Costero-Saint DA. Arthropod vectors and disease transmission: translational aspects. PLoS Negl Trop Dis. 2015;9:1-11.

41. Walker $\mathrm{T}$, Johnson PH, Moreira LA, Frentiu FD, Mcmeniman CJ, Leong YS, et al. The wMel Wolbachia strain blocks dengue and invades caged Aedes aegypti populations. Nature. 2011:476:450-3.

\section{Submit your next manuscript to BioMed Central and we will help you at every step:}

- We accept pre-submission inquiries

- Our selector tool helps you to find the most relevant journal

- We provide round the clock customer support

- Convenient online submission

- Thorough peer review

- Inclusion in PubMed and all major indexing services

- Maximum visibility for your research

Submit your manuscript at www.biomedcentral.com/submit

) Biomed Central 